\title{
Superconducting in Equal Molar NbTaTiZr-Based High-Entropy Alloys
}

\author{
Keo-Yuan Wu ${ }^{1}$, Swe-Kai Chen ${ }^{2 *}$, Jenn-Ming Wu ${ }^{1}$ \\ ${ }^{1}$ Department of Materials Science and Engineering, National Tsing Hua University, Hsinchu, Taiwan; ${ }^{2}$ Center for \\ Nanotechnology, Materials Science, and Microsystems (CNMM), National Tsing Hua University, Hsinchu, Taiwan
}

Correspondence to: Swe-Kai Chen, *skchen@mx.nthu.edu.tw

Keywords: HEAs, Superconducting, Matthias' Rule, e/a, NbTaTiZr-Based Alloys, Fe, Ge, Hf, Si, V Addition, Coherence Length, Electrical Carrier Density

Received: January 4, 2018 Accepted: March 27, 2018 Published: March 30, 2018

Copyright $\odot 2018$ by authors and Scientific Research Publishing Inc.

This work is licensed under the Creative Commons Attribution International License (CC BY 4.0).

http://creativecommons.org/licenses/by/4.0/

\section{(c) (1) Open Access}

\section{ABSTRACT}

Superconducting (SC) in equal molar NbTaTiZr-based high-entropy alloys (HEAs) that were added with $\mathrm{Fe}, \mathrm{Ge}, \mathrm{Hf}, \mathrm{Si}$, and/or $\mathrm{V}$ was observed. According to investigation on crystal structure, composition, and the relationship between critical temperature and e/a ratio, as indicated in Matthias' empirical rule, the main superconducting phase was that enriched with $\mathrm{Nb}$ and $\mathrm{Ta}$. The coherence length $(\xi)$ that was calculated from the carrier density reveals that $\xi$ value has the same order of magnitude of several hundreds of Angstroms as those binary $\mathrm{Nb}-\mathrm{Ti}$ and $\mathrm{Nb}-\mathrm{Zr}$ alloys showed.

\section{INTRODUCTION}

In the absence of an applied magnetic field, the electrical resistivity of a superconductor tends to zero upon cooling to a critical temperature, $T_{c}[1]$. As the temperature falls to $T_{o}$ and when the applied magnetic field is weaker than a critical field, $H_{\mathcal{o}}$ the magnetic flux is completely expelled from the superconductor, and a perfect diamagnetic state is formed [2]. Superconductors with these properties have been widely studied and applied in various applications.

Among elements in nature, $\mathrm{Nb}$ has the highest $T_{c}(9.25 \mathrm{~K})$. Conventional binary Nb-based alloys, such as $\mathrm{Nb}-\mathrm{Ti}(\sim 10 \mathrm{~K}), \mathrm{Nb}-\mathrm{Zr}(\sim 11 \mathrm{~K}), \mathrm{Nb}_{3} \mathrm{Sn}(18 \mathrm{~K})$, and $\mathrm{Nb}_{3} \mathrm{Ge}(23 \mathrm{~K})$, have even a higher $T_{c}$ than $\mathrm{Nb}$ [3]. These $T_{c}$ values are too low to enable the elements to be used under ambient conditions. Therefore, many attempts have been made to raise $T_{c}$ For example, researchers have used various heat treatments to control the formation of the superconducting phase of, e.g., $\mathrm{Nb}-\mathrm{Zr}$, [4]. In $\mathrm{Nb}-\mathrm{Ti}$ [5], as another example, third elements, $\mathrm{Al} \& \mathrm{Mo}$, have been used to generate 4.6 to 4.8 valence electrons per atom, e/a [6]. According to Matthias' empirical rule, the highest $T_{c}$ is obtained at the optimal e/a ratio for which the composition chooses, e.g., Mo, as a third element to reduce $T_{c}$ in $\mathrm{Nb}-\mathrm{Ti}$ [5]. However, adding $\mathrm{Al}$ promotes the formation of a phase of Nb-rich and increases $T_{c}$ [5]. However, the $T_{c}$ in ternary systems barely exceeds 
that of binary $\mathrm{Nb}$-Ti. Accordingly, the effectiveness of such approaches to increase $T_{c}$ is limited. Moreover, Matthias' empirical rule may not be applicable to multi-phase alloys. Difficulties still exist.

High-entropy alloys (HEAs) were proposed in 1995. These alloys were obtained by vacuum arc remelting $[7,8]$. The efforts to design and prepare HEAs have opened up yet another frontier in alloy research. They have been defined as alloys that contain principal elements of between five and 13 (inclusive), and have atomic concentrations of between five and 35 at\% (inclusive). Their main characteristics are high solubility, a large degree of lattice distortion, a large point lattice defect effect, slow atom diffusion, and a composite property effect (the so-called cocktail effect). Thermodynamically, the high-entropy effect, is a TS factor and a physical effect overcomes the enthalpy effect, which is an $\mathrm{H}$ factor and a chemical effect, increasing the solubility of each of the principal elements, promoting the generation of a random simple pseudo-unitary solid solution, such as with the A1, A2, or A3 crystal structure. This tendency to form a simple random solid solution thermodynamically enables the slow diffusion of atoms in the working processes, as occurs in thermal treatments. Hence, a nanostructure can be easily obtained. The high solubility of constituent elements makes the random solid solution highly ductile and hard. The HEA can simultaneously have the properties that conventional alloys with fewer multi-principal-element alloys cannot easily have simultaneously, such as heat resistance, anticorrosion, anti-oxidation, and precipitation hardening at high temperatures. Therefore, HEAs have great potential in the development of a range of applications [9]. HEAs have superior physical properties such as electrical conductivity, magnetic permeability, thermal conductivity, and hydrogen storage [10,11].

This study will explore superconductivity in HEAs. Multi-principal elements are added to modify their microstructure to study the effect of adding elements on the superconducting behaviors-zero electrical resistivity and diamagnetism-of simple pseudo-unitary BCC alloys [11]. The selected materials were equimolar NbTaTiZr-based HEAs. The results are compared with those for conventional superconductors.

\section{THEORY}

Superconductivity in conventional alloys has been microscopically explained by the BCS, which was proposed by Bardeen, Cooper, and Schrieffer in 1957 [12]. The central point of BCS is that electron Cooper pairs that are formed by electron-electron interaction under the influence of lattice phonon as the temperature falls below $T_{c}$ generate no electrical resistivity. The critical temperature for superconductivity is given by, [13]

$$
\begin{gathered}
T_{c}=1.14 \theta_{D} \exp \left(\frac{-1}{\lambda}\right), \\
\lambda=D\left(\varepsilon_{F}\right) V,
\end{gathered}
$$

where $\lambda$ is a superconducting coupling constant; $D\left(\varepsilon_{F}\right)$ is the electron density of states at the Fermi level; $\theta_{D} \quad\left(=\hbar \omega / k_{B}\right)$ is the Debye temperature; $k_{B}$ is Boltzmann's constant; $\omega$ is the frequency of lattice vibration, and $V$ is of the interaction between electrons in Cooper pairs. BCS applies only in weak coupling condition, i.e., $0<\lambda \ll 1$. In 1968, McMillan developed another expression for $T_{o}$ which considers the electron-phonon interaction, and is affected by both weak and strong couplings. This $T_{c}$ is given by [14]

$$
T_{c}=\frac{\theta_{D}}{1.45} \exp \left[\frac{-1.04(1+\lambda)}{\lambda-\mu^{*}(1+0.62 \lambda)}\right],
$$

where $\theta_{D}$ is Debye temperature; $\lambda$ is a coupling constant for the interaction between electrons and phonons, and $\mu^{*}$ is the repulsive coupling interaction constant in the interaction between electrons. McMillan also found that, for most metals, $\mu^{*} \approx 0.13$. Accordingly, Equation (3) can be simplified as:

$$
T_{c} \approx \frac{\theta_{D}}{1.45} \exp \left[\frac{-(1+\lambda)}{\lambda}\right],
$$




$$
\lambda=\frac{D\left(\varepsilon_{F}\right)\langle I\rangle^{2}}{M\langle\omega\rangle^{2}},
$$

where $I$ is the electron-phonon matrix element; $\omega$ is the phonon frequency in the metal lattice, and $M$ is the mass of a metal ion. From Equation (5), a larger $\lambda$, associated with higher $D\left(\varepsilon_{F}\right)$, a smaller $\omega$ or a smaller $\theta_{D}$ are associated with a higher $T_{c}$ For this reason, alloying elements are added. One paper on superconductivity in $\mathrm{Nb}-\mathrm{Cu}$ noted that, as the temperature falls to $\mathrm{T}_{\mathrm{i}}$, the silky network of the superconducting phase begins to allow the Cooper pairs to pass the alloy, generating superconductivity [15]. As the temperature falls further to $T_{f}$ if the coherence length is large enough, then the Cooper pairs generate an integrated superconducting path by the proximity effect. Therefore, determining whether superconducting HEAs exhibit the proximity effect and whether they are type II superconductors is essential. Some appropriate theories are used to calculate the coherence length $(\xi)$ and the Ginzburg-Landau parameter $(\kappa)$ from the carrier density [16]:

$$
n=\frac{I B}{V_{H} q t},
$$

where $n$ is the carrier density at $5 \mathrm{~K}$; $I$ is the current; $B$ is the applied magnetic field; $V_{H}$ is the Hall voltage; $t$ is the thickness of sample; and $q$ is the electric charge. After resistance vs. temperature (R-T) measurements were made to determine the electrical resistivity, the Drude model was used to calculate the electron relaxation time $\tau[16]$.

$$
\rho=\frac{m}{n q^{2} \tau}
$$

where $\rho$ is the $300 \mathrm{~K}$-resistivity; $m$ is the electron mass; $n$ is the carrier density; and $q$ is the electric charge. The Fermi velocity $v_{F}$ can also be obtained from the carrier density using Equation (8) [16].

$$
v_{F}=\left(\frac{\hbar}{m}\right)\left(3 \pi^{2} n\right)^{\frac{1}{3}} \text {. }
$$

The Fermi velocity and relaxation time can be used to determine the mean free path of the electrons using Equation (9) [16].

$$
\ell=v_{F} \tau .
$$

Equations ((10) \& (11)) yield the superconducting energy gap at $0 \mathrm{~K}, \Delta(0)$, and the intrinsic coherence length, $\xi_{0}$, respectively [17].

$$
\begin{gathered}
\Delta(0)=1.76 k_{B} T_{c} . \\
\xi_{0}=\frac{\hbar v_{F}}{\pi \Delta(0)},
\end{gathered}
$$

where $k_{B}$ is Boltzmann's constant; $T_{c}$ is the superconducting critical temperature; and $\hbar$ is Planck's constant over $2 \pi$.

In the mixed state of type II superconductors, each Abrikosov vortex has a core radius, $\xi$, at normal conducting (NC) state, surrounded by an outer radius, $\lambda$, of superconducting current. Each vortex carries a quantized flux, $\Phi_{0}=2.0678 \times 10^{-7} \mathrm{G} \mathrm{cm}^{2}$. As the applied magnetic field increases to the lower critical field, $H_{c 1}$, vortices arise. Hence, the flux $\Phi_{0}$ can be expressed as Equation (12) [16]:

$$
\phi_{0} \cong \pi \lambda^{2} H_{c 1} .
$$

The Ginzburg-Landau parameter, $\kappa$, can thus be obtained and used to identify the type of a superconductor by applying Equation (13) [3]. 


$$
\kappa=\frac{\lambda}{\xi} .
$$

The existence of superconductivity in HEAs was demonstrated and the coherence length is shown to be of the order of several hundred $\AA$, equal to that of binary $\mathrm{Nb}-\mathrm{Zr}$ and $\mathrm{Nb}$-Ti. Therefore, there is also a proximity effect like that in $\mathrm{Nb}-\mathrm{Zr}$ and $\mathrm{Nb}-\mathrm{Ti}$, is suggested to exist in superconducting HEAs. The calculated $\kappa$ value is 14.32 . This value, taken with results of magnetic measurements, reveals that superconducting HEAs are type II superconductors. Notably, Fe-containing NbTaTiZrFe has $T_{c}=6.87 \pm 0.03 \mathrm{~K}$. The highest obtained $T_{c}=8.46 \pm 0.02 \mathrm{~K}$ of NbTaTiZrGe [18] is close to that of element $\mathrm{Nb}$ of $9.25 \mathrm{~K}$, the two values are near. This result is not good enough, but, owing to the many degrees of freedom in the design of alloys, superconducting HEAs may open up a new avenue for research and applications.

\section{EXPERIMENTAL}

JEOL 5410 SEM-energy-dispersed X-ray spectroscope (SEM-EDS) was used to observe the microstructure and analyze the composition of alloys. Shimadzu XRD 6000 diffractometer was used in X-ray diffraction with $\mathrm{Cu}$-target (wavelength of $1.5405 \AA$ ), and operated at $30 \mathrm{kV}$ and $20 \mathrm{~mA}$. The scanning speed was $2 \%$ min with a $2 \theta$ range of 20 to 100 degrees.

To measure the transition temperature, specimens were cut in rectangles with a length of $8 \mathrm{~mm}$ and width of $3 \mathrm{~mm}$, and ground using \#400 SiC grinding paper to a thickness of $0.2 \mathrm{~mm}$. The four-point method was used to plot the resistivity vs. temperature, $R(T)$, curves at $4.2 \mathrm{~K}$ to $300 \mathrm{~K}$. The accuracy of the temperature measurement was better than $\pm 0.06 \mathrm{~K}$. The critical transition temperature, $T_{\mathcal{o}}$ is defined as the temperature at which the resistivity drops to $50 \%$ of the total resistivity. The interval of transition is defined by the formula $\Delta T_{c}=T_{c}(90 \%)-T_{c}(10 \%)$, where $90 \%$ and $10 \%$ indicate a drop in the resistivity to $90 \%$ and $10 \%$ of the original value, respectively, in the transition.

A SQUID setup was used to obtain both magnetic susceptibility vs. temperature, $\chi(T)$, and magnetization vs. applied magnetic field, $M(H) . \chi(T)$ at temperatures in the range $2 \mathrm{~K}$ to $300 \mathrm{~K}$ were obtained using the zero-field cooling (ZFC) method in applied magnetic fields of 100 Oe. Two fixed temperatures, $5 \mathrm{~K}$ and $300 \mathrm{~K}$, were used and the applied field was cyclically varied from 0 to 1 to 0 to -1 to $0 \mathrm{~T}$, and then back to $1 \mathrm{~T}$. The lower magnetic field, $H_{c 1}$, is the field that induces deviation from the diamagnetism $(\chi=$ $-1)$ line. When the demagnetization factor is considered, $H_{c 1}$ is given by Equation (14):

$$
H_{c 1}=\frac{H}{1-D},
$$

where $D$ is demagnetization factor. The upper critical field, $H_{c 2}$, is the smallest field that yields zero magnetization.

Hall measurements were made using a PPMS system (Quantum Design PPMS-9T) that was operated at $47 \mathrm{~Hz}, 50 \mathrm{~mA} \mathrm{AC}$, and from $9 \mathrm{~T}$ to $-9 \mathrm{~T}$ at $10 \mathrm{~K}$. Pt wires were attached using low-temperature Ag paste to the specimens to measure resistivity and to make the Hall measurements. The attachments were baked at $50^{\circ} \mathrm{C}$ to ensure an Ohmic contact. Carrier density was determined from the Hall voltage data.

Table 1 lists selected alloys, their phases, and their compositions. Both as-cast (C state) and homogenized ( $\mathrm{H}$ state) specimens were used. Some designations are given. They are equal-molar $\mathrm{C} 4$ \& $\mathrm{H} 4$ for NbTaTiZr, C5 \& H5 for NbTaTiZrHf, C6 \& H6 for NbTaTiZrHfV, and C7 \& H7 for NbTaTiZrHfVMo. All specimens were melted and cast in a remelter in an atmosphere of Ar using a water-cooled $\mathrm{Cu}$ hearth. As-cast specimens were melted once, and then turned and melted three more times to ensure their homogeneity. Samples were homogenized in a quartz tube that was filled with argon gas at $1100^{\circ} \mathrm{C}$ for $1 \mathrm{~d}$ and then quenched in water.

Figure 1 shows back-scattered electron images (BEI). Table 1 lists energy-dispersed X-ray spectroscopic (EDS) data for both as-cast and homogenized specimens. White Nb-Ta-rich dendrites and black $\mathrm{Ti}$-Zr-rich interdendrites are observed in $\mathrm{C} 4$, and white $\mathrm{Nb}$-Ta-rich dendrites and black $\mathrm{Zr}$-Hf-rich interdendrites are observed in both C5 and C6. XRD patterns (Figure 2) reveal that alloys C4 to C6 are simple 

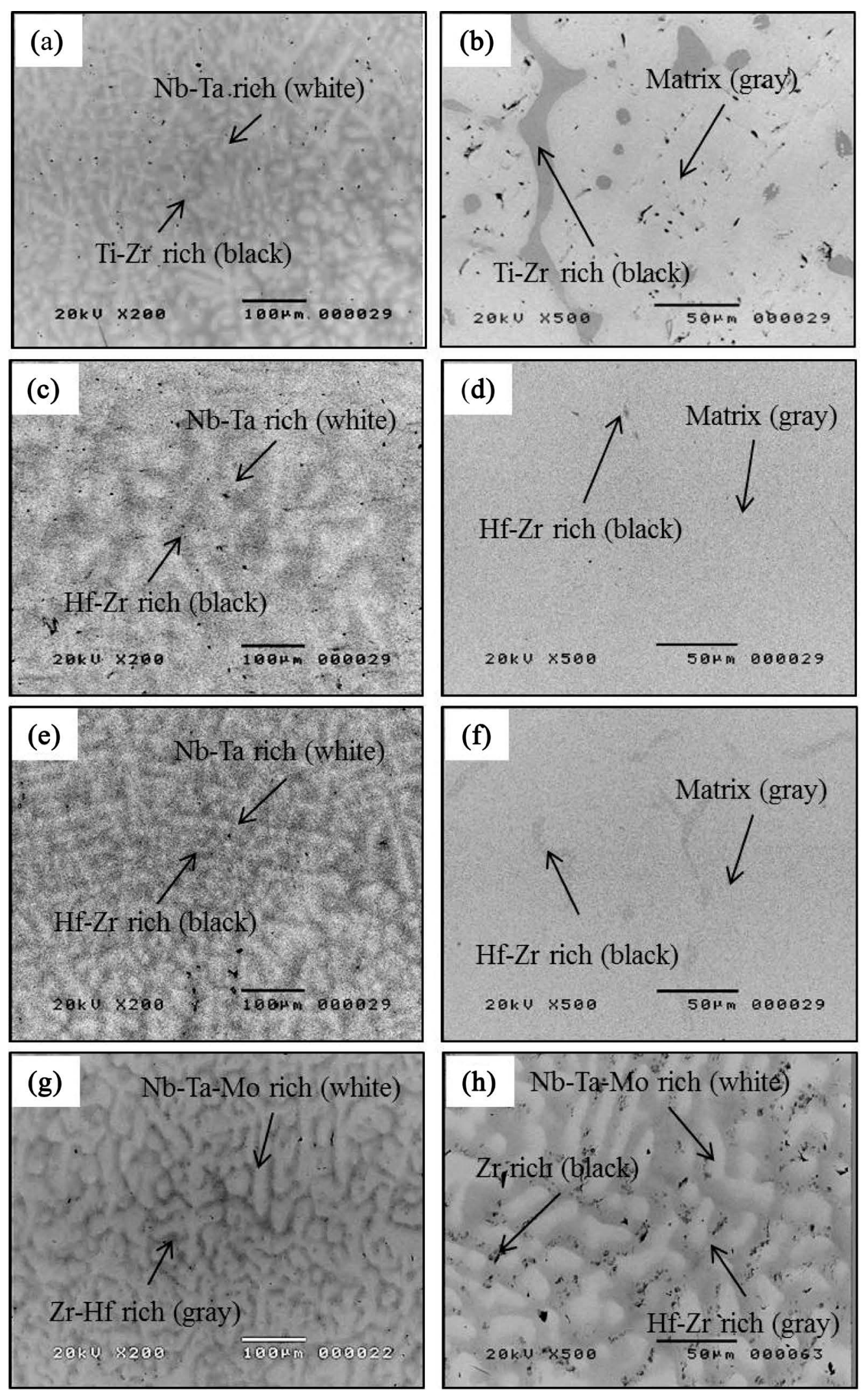

Figure 1. The back-scattered electron images (BEI) for as-cast and homogenized HEAs. (a) C4, (b) $\mathrm{H} 4$, (c) C5, (d) H5, (e) C6, (f) H6, (g) C7, (h) H7. 
Table 1. Compositions (at\%) of selected alloy materials and their phases.

\begin{tabular}{|c|c|c|c|c|c|c|c|c|}
\hline Sample & Phase & $\mathrm{Nb}$ & $\mathrm{Ta}$ & $\mathrm{Ti}$ & $\mathrm{Zr}$ & $\mathrm{Hf}$ & $\mathrm{V}$ & Mo \\
\hline \multirow{2}{*}{$\mathrm{C} 4$} & Dendrite (Nb-Ta rich) & 25.2 & 30.7 & 21.3 & 22.8 & & & \\
\hline & Interdendrite (Ti-Zr rich) & 19.4 & 18.1 & 26.3 & 36.2 & & & \\
\hline \multirow{2}{*}{$\mathrm{H} 4$} & Matrix & 26.1 & 25.1 & 23.4 & 25.4 & & & \\
\hline & Interdendrite (Ti-Zr rich) & 21.1 & 16.3 & 24.3 & 38.3 & & & \\
\hline \multirow{2}{*}{ C5 } & Dendrite (Nb-Ta rich) & 22.1 & 26.3 & 16.6 & 15.5 & 19.5 & & \\
\hline & Interdendrite (Zr-Hf rich) & 16.7 & 14.3 & 20.4 & 25.2 & 23.4 & & \\
\hline \multirow{2}{*}{ H5 } & Matrix & 19.8 & 18.9 & 20.8 & 18.7 & 21.8 & & \\
\hline & Interdendrite (Zr-Hf rich) & 16.6 & 18.2 & 20.6 & 21.5 & 23.1 & & \\
\hline \multirow{2}{*}{ C6 } & Dendrite (Nb-Ta rich) & 21.5 & 18.1 & 15.9 & 14.4 & 16.6 & 13.5 & \\
\hline & Interdendrite (Zr-Hf rich) & 16.8 & 11.5 & 16.3 & 19.7 & 18.3 & 17.4 & \\
\hline \multirow{2}{*}{ H6 } & Matrix & 16.3 & 15.7 & 16.9 & 17.1 & 17.5 & 16.5 & \\
\hline & Interdendrite (Hf-V rich) & 7.4 & 8.4 & 7.1 & 26.3 & 22.8 & 27.0 & \\
\hline \multirow{3}{*}{$\mathrm{C7}$} & Dendrite (Nb-Ta-Mo rich) & 17.1 & 15.2 & 13.5 & 11.8 & 13.9 & 12.9 & 15.6 \\
\hline & Interdendrite (Zr-Hf rich) & 11.4 & 6.8 & 16.1 & 22.7 & 19.5 & 14.6 & 8.9 \\
\hline & Inner-Interdendrite ( $\mathrm{Zr}-\mathrm{Hf}$ rich) & 9.6 & 5.8 & 14.9 & 26.9 & 20.5 & 14.0 & 8.3 \\
\hline \multirow{3}{*}{$\mathrm{H7}$} & Dendrite (Nb-Ta-Mo rich) & 19.8 & 22.6 & 11.7 & 6.4 & 9.5 & 11.1 & 18.9 \\
\hline & Interdendrite (Zr-Hf rich) & 13.1 & 10.5 & 14.3 & 18.7 & 17.2 & 14.1 & 12.1 \\
\hline & Inner-Interdendrite ( $\mathrm{Zr}$ rich) & 12.6 & 10.9 & 15.8 & 21.9 & 13.9 & 15.3 & 9.6 \\
\hline
\end{tabular}
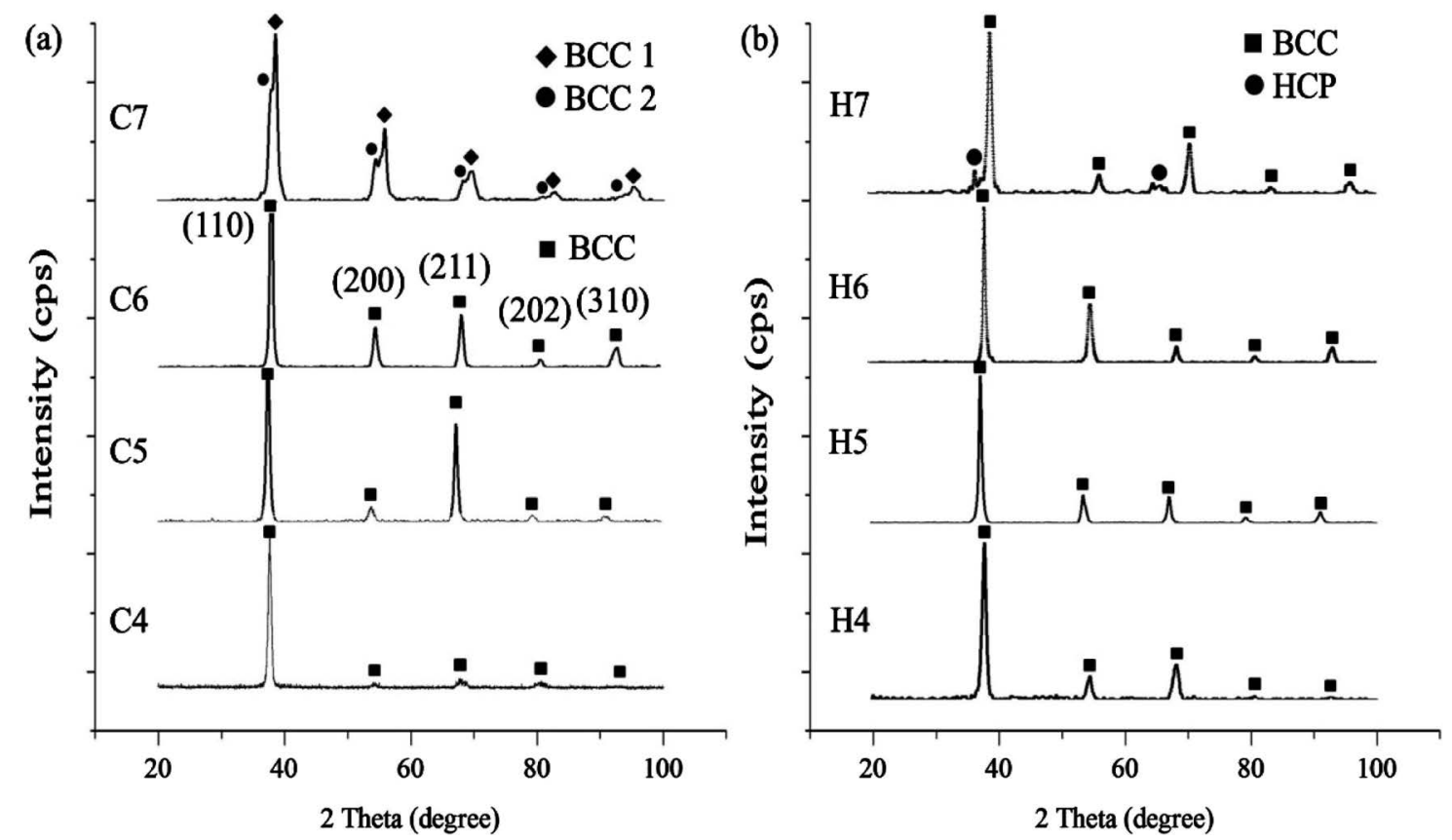

Figure 2. XRD diffraction patterns at room temperature. (a) As-cast HEAs, (b) homogenized HEAs. 
pseudo-unitary A2 random BCC solid solutions with lattice parameters of $3.3728 \AA$, $3.4051 \AA$, and 3.3567 $\AA$, respectively. $\mathrm{H} 4$ to $\mathrm{H} 6$ have a matrix, grown from the dendrites, and they contain less interdendrite than the C4 to C6, respectively. The crystal structures and lattice parameters of the as-homogenized samples are almost the same as those of the as-cast samples. Alloys with added Mo, Fe, Ge, Hf, Si, V, and/or W are used to examine the dependence of $T_{c}$ on the e/a ratio and to verify Matthias' empirical rule. These are NbTaMoW, NbTaMoVW, NbTaTiZrHfVMo (C7 BCC1 \& H7 BCC with lattice parameters of 3.2840 \& $3.2711 \AA$, respectively), NbTaTiZrFe, NbTaTiZrGe, NbTaTiZrGeV, NbTaTiZrSiV, NbTaTiZrSiGe, and NbTaTiZrSiGeV.

\section{RESULTS AND DISCUSSION}

\subsection{Measurement of Transition Temperatures}

Figure 3 plots the electrical resistivity as a function of temperature from $4.2 \mathrm{~K}$ to $300 \mathrm{~K}$ in the absence of a magnetic field. C4 to C6 have a superconducting transition at $8.29 \pm 0.04 \mathrm{~K}, 7.12 \pm 0.02 \mathrm{~K}$, and $5.09 \pm$ $0.03 \mathrm{~K}$ (Figure 3(a) to Figure 3(c)), respectively, while C7 has no transition in the temperature interval in which measurements are made (Figure $3(\mathrm{~d})$ ). The transition temperature decreases as $\mathrm{Hf}$ and $\mathrm{V}$ are added to NbTaTiZr (C4) to form equimolar $\mathrm{C} 5(\mathrm{C} 4+\mathrm{Hf})$ and $\mathrm{C} 6(\mathrm{C} 5+\mathrm{V})$ alloys. Following the addition of Mo to $\mathrm{C} 6$ to form $\mathrm{C} 7$, the alloy undergoes no transition between $4.2 \mathrm{~K}$ and $300 \mathrm{~K}$. Therefore the dilution of $\mathrm{C} 4$ by Hf, $\mathrm{V}$ or Mo reduces the transition temperature. The critical temperatures or $T_{c}$ values of $\mathrm{H} 4$ to $\mathrm{H} 6$ samples are, respectively, $8.27 \pm 0.03 \mathrm{~K}, 6.69 \pm 0.04 \mathrm{~K}$, and $4.96 \pm 0.04 \mathrm{~K}$, which are below the values for the corresponding as-cast alloys (Figure 4). Like C7, alloy H7 exhibits no transition in the range 4.2 to 300 $\mathrm{K}$.
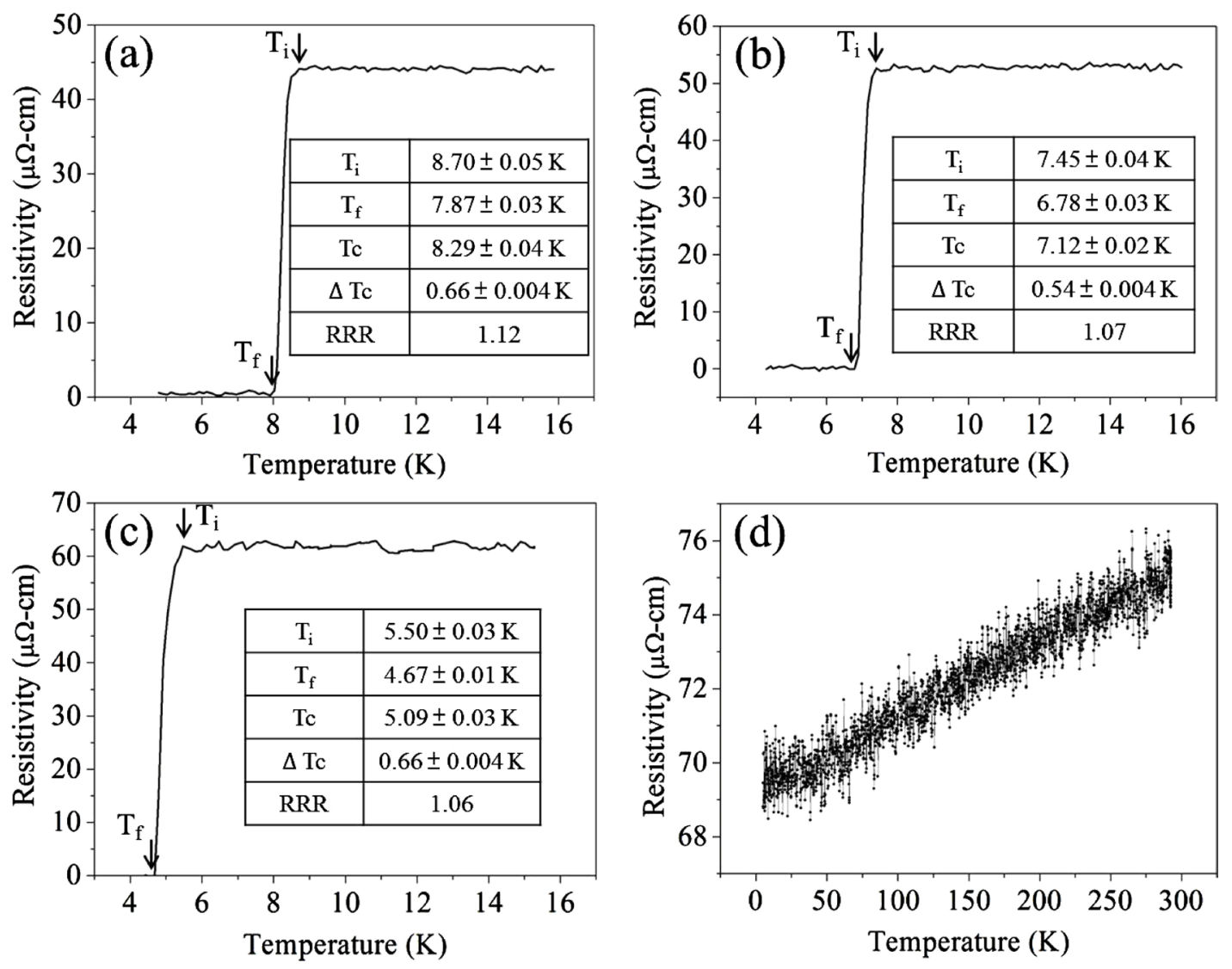

Figure 3. The electrical resistivity as a function of temperature from 4.2 to $300 \mathrm{~K}$ in the absence of a magnetic field for as-cast HEAs. (a) C4, (b) C5, (c) C6, (d) C7. 

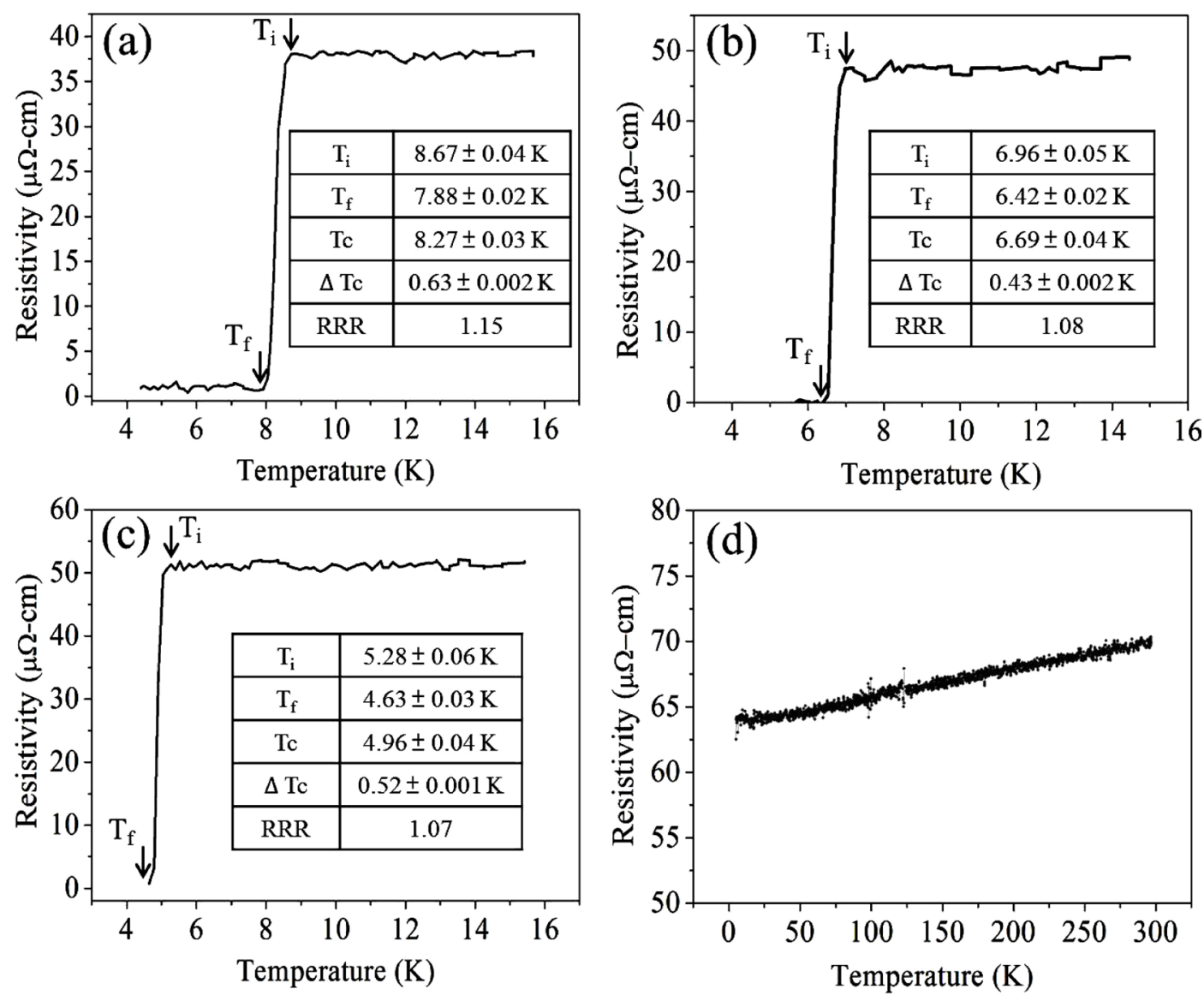

Figure 4. The electrical resistivity as a function of temperature from 4.2 to $300 \mathrm{~K}$ in the absence of a magnetic field for homogenized HEAs. (a) H4, (b) H5, (c) H6, (d) H7.

\subsubsection{Dependence of Critical Temperature on Microstructure}

The major difference between the microstructures of $\mathrm{C} 4$ to $\mathrm{C} 6$ and that of $\mathrm{C} 7$ is that the dendrite phase of the former three alloys ( $\mathrm{C} 4$ to $\mathrm{C6}$ ) is $\mathrm{Nb}$-Ta rich, while that of the latter (C7) is Nb-Ta-Mo rich. Both the formation of the Nb-Ta-rich phase in $\mathrm{C} 4$ to $\mathrm{C} 6$ and that of the $\mathrm{Nb}-\mathrm{Ta}$-Mo-rich phase in $\mathrm{C} 7$ are attributable to the enthalpy of formation of the elements in the alloys, which are not the focus of this paper. With respect to composition, the total atomic percentages of $\mathrm{Nb}+\mathrm{Ta}$ in the white phases of the $\mathrm{C} 4$ to C7 alloys are, respectively, 55.9, 48.4, 39.6, and 32.3 at\%. Their corresponding $T_{c}$ values are $8.29 \pm 0.04 \mathrm{~K}$, $7.12 \pm 0.02 \mathrm{~K}, 5.09 \pm 0.03 \mathrm{~K}$, and below $4.2 \mathrm{~K}$, respectively. The $T_{c}$ values are clearly related to the total amounts of $\mathrm{Nb}+\mathrm{Ta}$ in the $\mathrm{C} 4$ to C7 alloys. Other alloys, such as NbTaTiZrGe and NbTaTiZrFe, have 80+ at\% and approximately $75 \mathrm{at} \%$ of $\mathrm{Nb}+\mathrm{Ta}$ in their white phases, respectively, with $\mathrm{T}_{\mathrm{c}}$ values of 8.46 and $6.87 \mathrm{~K}$. Similar observations have been made of the $\mathrm{Nb}-\mathrm{Zr}$ system [4] by authors who applied thermal treatments to raise the $\mathrm{Nb}$ content in the $\beta$ matrix phase, and observed higher $\mathrm{T}_{\mathrm{c}}$. In this study, homogenization reduced the total $\mathrm{Nb}+\mathrm{Ta}$ contents in the $\mathrm{Nb}$-Ta-rich matrix phase, decreasing $\mathrm{T}_{\mathrm{c}}$ to less than that of the as-cast state.

The above SC HEAs, like conventional SC alloys, undergo a transition under an interval of temperatures. The width of the interval, $\Delta T$, depends on the purity and the defect concentration of the sample. Homogenized samples have a more uniform composition and contain fewer defects, so they have a smaller $\Delta T$ (Figure 4).

\subsection{2. $T_{c}$ as a Function of Electron-to-Atom Ratio $(e / a)$}

Some papers have discussed Matthias' empirical rule, concerning the relationship between $T_{c}$ and $e / a$ 
$[6,17,19]$. Matthias' empirical rule states that $T_{c}$ depends on $e / a$ in a particular periodical manner. When e/a ratio are in the range of 4.6 to 4.8 , there occurs higher $T_{c}$ The e/a of each HEA is calculated herein by giving that the e/a values for the IVB, VB, and VIB elements in the Periodic Table of Elements are 4, 5, and 6 , respectively, and the calculated e/a values are compared with $T_{c}$ The results of the comparison are listed in Table 2 and plotted in Figure 5 for comparison with the empirical rule. C4, C5, NbTaMoW and

Table 2. The calculated e/a and $T_{c}$ values of as-cast HEAs.

\begin{tabular}{|c|c|c|c|c|}
\hline As-cast alloys & $T_{c}(\mathrm{~K})$ & Overall e/a & Phases & $e / a$ \\
\hline \multirow{2}{*}{ NbTaTiZr (C4) } & \multirow{2}{*}{8.29} & \multirow{2}{*}{4.50} & White (Nb-Ta rich) & 4.559 \\
\hline & & & Black (Ti-Zr) & 4.375 \\
\hline \multirow{2}{*}{ NbTaTiZrHf (C5) } & \multirow{2}{*}{7.12} & \multirow{2}{*}{4.40} & White (Nb-Ta rich) & 4.484 \\
\hline & & & Black (Hf-Zr rich) & 4.310 \\
\hline \multirow{3}{*}{ NbTaTiZrHfV (C6) } & \multirow{2}{*}{5.09} & \multirow{2}{*}{4.50} & White (Nb-Ta rich) & 4.531 \\
\hline & & & Black (Hf-Zr rich) & 4.401 \\
\hline & \multirow{3}{*}{ N.A. } & \multirow{3}{*}{4.70} & White (Nb-Mo-Ta rich) & 4.764 \\
\hline \multirow[t]{3}{*}{ NbTaTiZrHfVMo (C7) } & & & Gray (Hf-Zr rich) & 4.506 \\
\hline & & & Black (Hf-Zr rich) & 4.460 \\
\hline & \multirow{3}{*}{6.87} & \multirow{3}{*}{5.20} & White (Nb-Ta rich) & 4.884 \\
\hline \multirow[t]{3}{*}{ NbTaTiZrFe } & & & Gray (Zr-Fe rich) & 5.474 \\
\hline & & & Black (Ti-Zr rich) & 4.905 \\
\hline & \multirow{3}{*}{8.46} & \multirow{3}{*}{4.40} & White (Nb-Ta rich) & 4.835 \\
\hline \multirow[t]{2}{*}{ NbTaTiZrGe } & & & Gray (Ti rich) & 4.299 \\
\hline & & & Black (Ge rich) & 4.301 \\
\hline \multirow{3}{*}{ NbTaTiZrGeV } & \multirow{3}{*}{8.40} & \multirow{3}{*}{4.50} & White (Nb-Ta rich) & 4.834 \\
\hline & & & Gray (Ti-V rich) & 4.626 \\
\hline & & & Black (Zr-Ge rich) & 4.144 \\
\hline \multirow{3}{*}{ NbTaTiZrSiV } & \multirow{3}{*}{4.29} & \multirow{3}{*}{4.50} & White (Nb-Ta rich) & 4.675 \\
\hline & & & Gray (Ti-V rich) & 4.549 \\
\hline & & & Black (Zr-Si rich) & 4.314 \\
\hline \multirow{3}{*}{ NbTaTiZrSiGe } & \multirow{3}{*}{7.40} & \multirow{3}{*}{4.33} & White (Nb-Ta rich) & 4.779 \\
\hline & & & Gray (Nb-Zr-Ge rich) & 4.303 \\
\hline & & & Black (Si rich) & 4.213 \\
\hline \multirow{3}{*}{ NbTaTiZrSiGeV } & \multirow{3}{*}{ N.A.* } & \multirow{3}{*}{4.43} & White (Ta-V rich) & 4.758 \\
\hline & & & Gray (V rich) & 4.747 \\
\hline & & & Black (Si rich) & 4.257 \\
\hline \multirow{2}{*}{ NbTaMoW } & 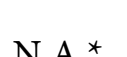 & & White (Ta-W rich) & 5.524 \\
\hline & N.A." & 5.5 & Black (Nb-Mo rich) & 5.472 \\
\hline NhTaMoWV & $\mathrm{N} A$ * & 54 & White (Ta-W rich) & 5.450 \\
\hline No i anvo va & N.R. & 3.4 & Black (Nb-V rich) & 5.288 \\
\hline
\end{tabular}

*"N.A." means $T_{c}<4.2 \mathrm{~K}$. 

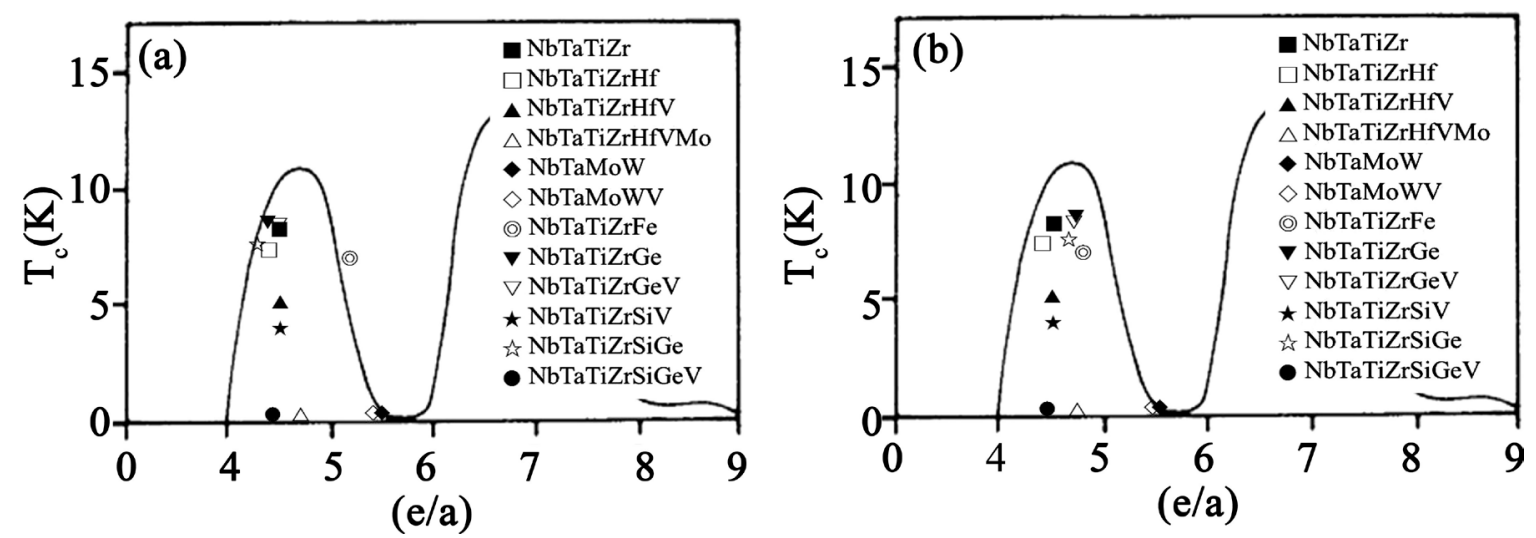

Figure 5. $T_{c}$ values vs. e/a values for HEAs (Matthias' curve after H.R. Khan). (a) e/a values for overall average composition, (b) e/a values for white-phase composition.

NbTaMoWV qualitatively satisfy the empirical rule (Figure 5(a)). C6, C7, NbTaTiZrFe, NbTaTiZrSiV, and $\mathrm{NbTaTiZrSiGeV}$ show point deviation from the empirical curve. As the e/a ratio increases from four to five, $T_{c}$ increases; as it increases from five to six, $T_{c}$ falls to below $4.2 \mathrm{~K}$. In an alloy with multiphase or heterogeneous composition, the ratio seems not to be the only indicator of $T_{c}$ [5]. Values of e/a calculated from the composition of the white phase are presented in Table 2. Both the Nb-Ta-rich "white" phase (C4, C5, C6, NbTaTiZrFe, NbTaTiZrGe, NbTaTiZrGeSi, NbTaTiZrGeV, and NbTaTiZrSiV) and the $\mathrm{Nb}$-Ta-Mo-rich "white" phase (C7) have e/a ratio that is close to the 4.6-4.8-peak in the empirical curve. Therefore, the white phase is concluded to be the SC phase with the highest $T_{c}$ The e/a ratio for $\mathrm{A} 2-\mathrm{NbTaMoW}$ and $\mathrm{A} 2-\mathrm{NbTaMoVW}$ are in the range from five to six, so these alloys have lower $T_{c}$ The e/a values that are calculated from the compositions of the white phases in alloys C4, C5, C6, NbTaTiZrFe, $\mathrm{NbTaTiZrGe}$, NbTaTiZrGeSi, NbTaTiZrGeV, and NbTaTiZrSiV "match" the empirical curve (Figure 5(b)) with the exception that although e/a is in the range 4.6 to 4.8 for $\mathrm{C} 7$ and NbTaTiZrGeSiV, their $T_{c}$ values are lower than $4.2 \mathrm{~K}$ and so could not be observed in this experiment. Interestingly, fewer added elements in the alloys $\mathrm{C} 4$ to $\mathrm{C} 7$ correspond to higher $T_{c}$ values. This fact motivates a test of the effect of the lattice distortion (related to number of elements $n$ ) on $T_{c}$. The root mean square (RMS), as in Equation (15), is used to calculate the degree of lattice distortion. Both lattice expansion and compression are considered because of the square term in this equation.

$$
\mathrm{RMS}=\sqrt{\frac{\sum_{i=1}^{n} x_{i}^{2}}{n}},
$$

where $n$ is the number of elements in the alloy and $x_{i}$ is the difference between each atomic radius and the mean or equivalent atomic radius that is calculated from the lattice parameter. Since the main lattice structure herein is a pseudo-unitary A2 structure [11], Equation (16) can be utilized to obtain the mean or equivalent atomic radius.

$$
4 R=\sqrt{3} a,
$$

where " $R$ " is the mean radius and " $a$ " is the lattice parameter. Table 3 presents the values of $R$, $a$, and RMS of the HEAs in this study. As $n$ increases, the RMS also increases. This fact, along with the determined values of $T_{c}$, reveals that for SC HEAs with a particular e/a, a lower RMS (or lower $\mathrm{n}$ ) corresponds to a higher $T_{c}$ This result is the same as obtained for SC FCC alloys, obtained by Mota and Hoyt [20], who studied alloys of precious metals to which had been added various impurity contents and found that for a fixed $e / a$ value, a higher impurity content caused greater distortion of the FCC lattice and a lower $T_{\mathcal{o}}$ regardless of whether the distortion was expansive or compressive. The concentration of defects can be evaluated from the RRR (relative residual resistivity) (Figure 3 and Figure 4). As RRR approaches unity, the 
Table 3. The values of lattice parameter $(\AA), R(\AA)$, and RMS/R (\%) of as-cast HEAs.

\begin{tabular}{cccc}
\hline As-cast alloys \& alloy designations & $\mathrm{R}(\AA)$ & Lattice parameter $(\AA)$ & $\mathrm{RMS} / \mathrm{R}(\%)$ \\
\hline NbTaTiZr (C4) & 1.4605 & 3.3728 & 4.67 \\
NbTaTiZrHf (C5) & 1.4744 & 3.4051 & 4.97 \\
NbTaTiZrHfV (C6) & 1.4535 & 3.3567 & 6.12 \\
NbTaTiZrHfVMo (C7) & 1.4343 & 3.2988 & 6.48 \\
\hline
\end{tabular}

effect of the defect concentration on the resistivity becomes stronger. The RRR values of HEAs in this study are near unity, and as $\mathrm{n}$ increases, the RRR initially declines, and then increases. However, RRR does not significantly affect $\mathrm{T}_{\mathrm{c}}$ in this study.

McMillan proposed Equations ((4) \& (5)) and noted that as $D\left(\varepsilon_{F}\right)$ increases and $\theta_{D}$ decreases, $\lambda$ increases, increasing $T_{c}$ Ramakrishnan et al. found that ternary $\mathrm{Nb}_{50} \mathrm{Ti}_{30} \mathrm{M}_{20}(\mathrm{M}=\mathrm{Mo}, \mathrm{Pt}, \mathrm{Pd}, \mathrm{V}$, and $\mathrm{Ru})$ alloys have a higher $\theta_{D}$ than does $\mathrm{Nb}_{60} \mathrm{Ti}_{40}$, and so they have lower values of $T_{c}$ [21]. An increase of $\mathrm{n}$ in HEAs has been suggested to increase $\theta_{D}$, and reduce $T_{c}$ This $\theta_{D}$ factor is responsible for the fact that alloys $\mathrm{C} 7$ and NbTaTiZrGeSiV have lower values of $T_{c}$ The effect of $D\left(\varepsilon_{F}\right)$ will be discussed.

The SC of HEAs is concluded to depend not only on the distribution of $\mathrm{Nb}+\mathrm{Ta}$ in the SC phase, but also on the lattice distortion and the consequent change in $\theta_{D}$.

\subsection{Magnetization Measurement}

\subsection{1. $\chi(\mathrm{T})$ Curve}

SQUID measurements of as-cast alloys were made to study the magnetization of SC HEAs. Figure 6 plots $\chi(\mathrm{T})$ at $100 \mathrm{Oe}$ for $\mathrm{C} 4$ to C7. From Figure 6, diamagnetism occurs in C4, C5, C6 and C7 as the temperature falls to $7.86 \pm 0.02 \mathrm{~K}, 6.37 \pm 0.03 \mathrm{~K}, 4.96 \pm 0.02 \mathrm{~K}$, and $3.49 \pm 0.03 \mathrm{~K}$, respectively. These first three temperatures are slightly lower than the corresponding $T_{c}$ values $(8.29 \pm 0.04,7.12 \pm 0.02$, and $5.09 \pm$ 0.03 ) that were obtained from $R(T)$ in Figures 3(a)-(c), respectively, indicating that an applied magnetic field reduces $T_{c}$ Furthermore, at temperatures of greater than $T_{\mathcal{o}}$ at which the diamagnetism disappears and paramagnetism occurs, the $\chi$ values for $\mathrm{C} 4, \mathrm{C} 5, \mathrm{C} 6$ and $\mathrm{C} 7$ are independent of temperature during cooling. Therefore, the magnetism of $\mathrm{C} 4$ to $\mathrm{C} 7$ in their normal conducting (NC) state is Pauli paramagnetism.

\subsection{2. $\mathrm{M}(\mathrm{H})$ Curve}

Figure $7(\mathrm{a})$ plots $\mathrm{M}(\mathrm{H})$ for $\mathrm{C} 4$ and $\mathrm{C} 5$ at $5 \mathrm{~K}$, and that for $\mathrm{C} 6$ at $2 \mathrm{~K}$. These alloys are typical type II SCs. When the applied magnetic field is less than $H_{c 1}$ the SC is in the perfect diamagnetic state. The values of $H_{c 1}$ are obtained from Equation (14). However, since too few data points are obtained in a weak magnetic field to obtain a precise value of $H_{c 1}$, the $H_{c 1}$ values in this study have a slightly large standard deviation. The $H_{c 1}$ values of alloys $\mathrm{C} 4$ and $\mathrm{C} 5$ at $5 \mathrm{~K}$, and of $\mathrm{C} 6$ at $2 \mathrm{~K}$, are $664 \pm 128,77 \pm 22$, and $440 \pm 155 \mathrm{Oe}$, respectively. As the number of alloying elements increases, $H_{c 1}$ falls. However, C6 has a larger $H_{c 1}$ than $\mathrm{C} 5$ because $H_{c 1}$ is a function of temperature, increasing as the temperature falls. As the applied magnetic field increases above $H_{c 1}$, the \% dia-magnetization falls, reaching zero at $H_{c 2}$. The SC-NC mixed state exists between $H_{c 1}$ and $H_{c 2}$. In this experiment, the field applied to C4 and C5 was limited to only 1 Tesla (T), and since the $H_{c 2}$ values of these alloys exceed $1 \mathrm{~T}, H_{c 2}$ was not observed. However, extrapolation yielded an $H_{c 2}$ in $\mathrm{C} 4$ and $\mathrm{C} 5$ at $5 \mathrm{~K}$ of approximately $1.38 \mathrm{~T}$ and $1.85 \mathrm{~T}$, respectively. Since the maximum field that could be applied to C6 was $4 \mathrm{~T}$, its $H_{c 2}$ could be obtained $1.93 \mathrm{~T}$ at $2 \mathrm{~K}$.

$\mathrm{C} 4$ to $\mathrm{C} 7$ in normal state show paramagnetism, as can be seen in $\mathrm{M}(\mathrm{H})$ at $300 \mathrm{~K}$ (Figure 7(b)). Because $\chi(T)$ in normal state is independent of T, C4 to C7 are Pauli paramagnetic. Hence, the electron density of state at the Fermi level, $D\left(\varepsilon_{F}\right)$, can be calculated from this Pauli susceptibility, $\chi_{p}$, by Equation (17) [22]: 


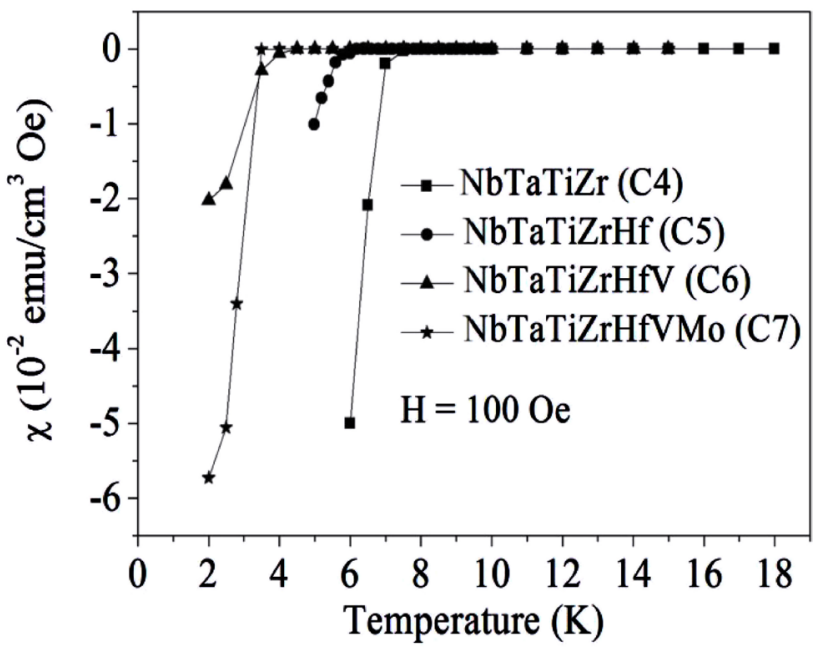

Figure 6. The magnetization as a function of temperature curves, $\chi(T)$, for as-cast HEAs.
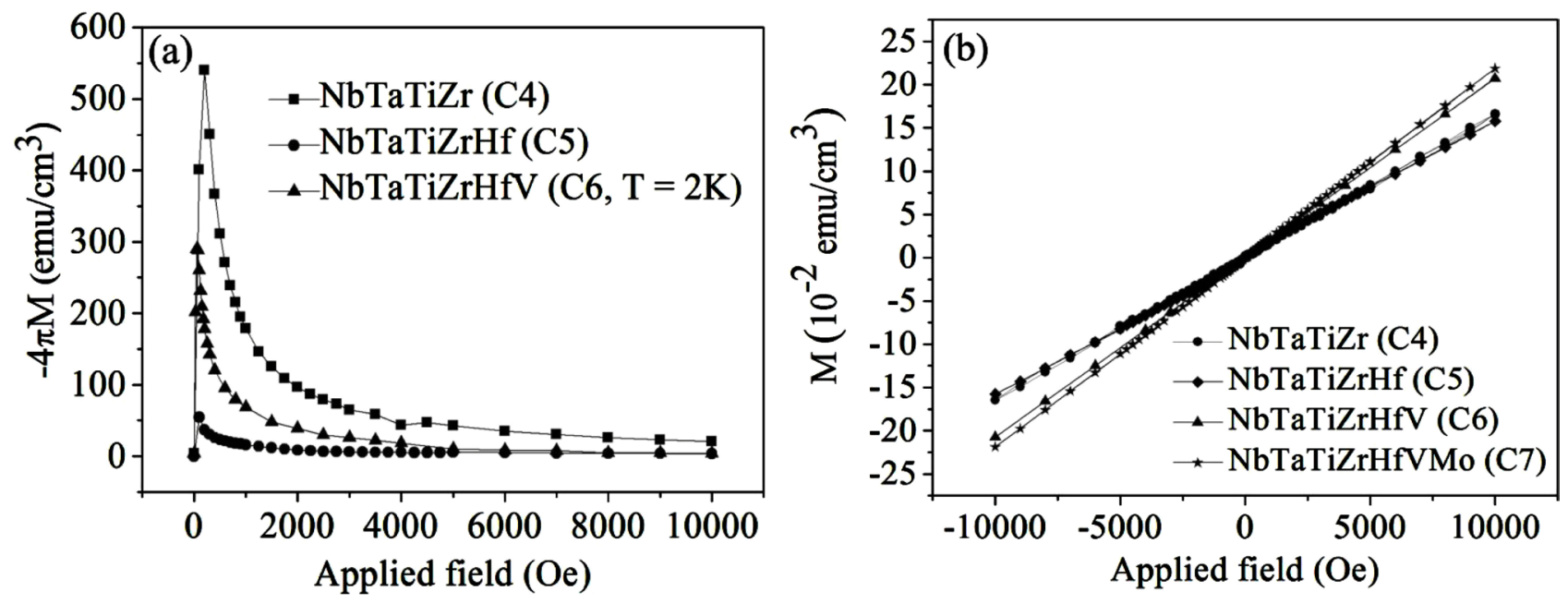

Figure 7. The magnetization as a function of magnetic fields, (M-H), for as-cast HEAs. (a) at $T=5$ $\mathrm{K}$, (b) at $T=300 \mathrm{~K}$.

$$
\chi_{p}=\mu_{B}^{2} \times D\left(\varepsilon_{F}\right),
$$

where $\mu_{B}$ is the Bohr magneton. Values of $\chi_{p}$ for $\mathrm{C} 4$ to $\mathrm{C} 7$ are, respectively, $1.65 \times 10^{-5}, 1.56 \times 10^{-5}, 2.07 \times$ $10^{-5}$, and $2.18 \times 10^{-5} \mathrm{emu} / \mathrm{cm}^{3} \cdot$ Oe. Clearly, C7 has the largest $\chi_{p}$ and largest $D\left(\varepsilon_{F}\right)$, since $\chi_{p}$ is proportional to $D\left(\varepsilon_{F}\right)$. By Equations $((4) \&(5))$, higher $D\left(\varepsilon_{F}\right)$ and smaller $\theta_{D}$ yield a higher $T_{\mathcal{O}}$ C7 has the largest $D\left(\varepsilon_{F}\right)$ and the lowest $T_{\mathcal{O}}$ suggesting that it has the largest $\theta_{D}$.

Figure 8 plots the $H_{c 2}$ values of some typical conventional SC alloys and SC HEAs. The equimolar NbTaTiZr-based alloys have lower $H_{c 2}$ values than do conventional A-15 alloys. However, the design of HEAs benefits from many degrees of freedom, extensive SC-HEA research is possible.

\subsection{Hall Measurements}

The calculated carrier density in $\mathrm{C} 5$ at $10 \mathrm{~K}$ is of the order of $10^{22} \mathrm{~cm}^{-3}$-similar to that of pure metals. From the carrier density, electrical conductivity data are calculated (Table 4). The Fermi velocities of HEAs are similar to those of pure metals. Since the lattice distortion is greater in HEAs, their 300 $\mathrm{K}$-resistivity is also higher, and the mean free path 1 of carriers is smaller. The calculated 1 values satisfy the 


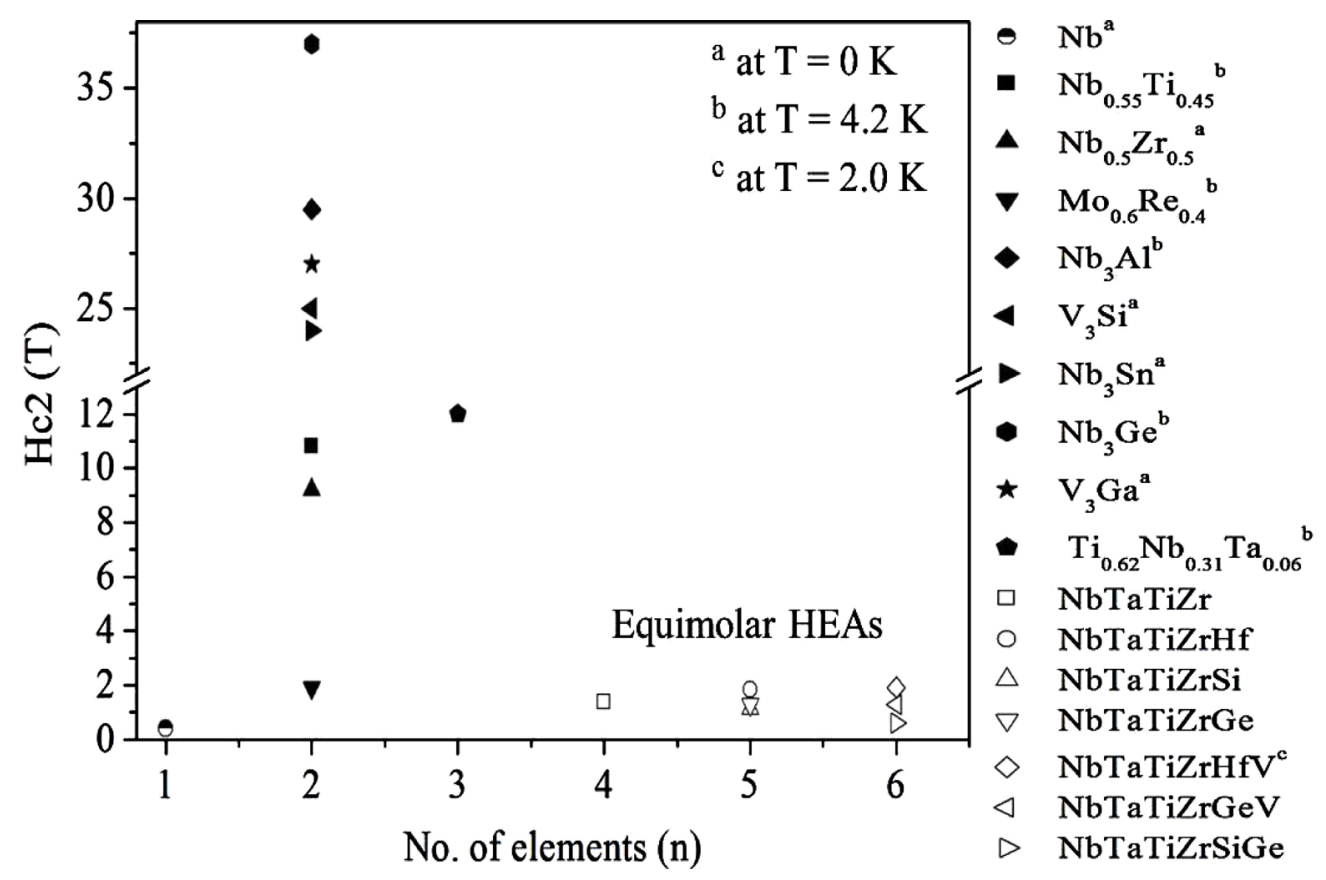

Figure 8. The $H_{c 2}$ values for conventional alloys $(0 \mathrm{~K} \& 4.2 \mathrm{~K})$ and equimolar HEAs $(2 \mathrm{~K} \& 5 \mathrm{~K})$.

Table 4. Electrical conductivity data of alloy C5.

\begin{tabular}{ccccccccc}
\hline $\begin{array}{c}\rho_{10-\mathrm{K}} \\
(\mu \Omega-\mathrm{cm})\end{array}$ & $\begin{array}{c}\rho_{300-\mathrm{K}} \\
(\mu \Omega-\mathrm{cm})\end{array}$ & $\begin{array}{c}N_{10-\mathrm{K}} \\
\left(10^{22} \mathrm{~cm}^{-3}\right)\end{array}$ & $\begin{array}{c}T_{c} \\
(\mathrm{~K})\end{array}$ & $\begin{array}{c}\tau \\
\left(10^{-15} \mathrm{~s}\right)\end{array}$ & $\begin{array}{c}v_{F} \\
\left(10^{8} \mathrm{~cm} / \mathrm{s}\right)\end{array}$ & $\begin{array}{c}\ell \\
\left(10^{-7} \mathrm{~cm}\right)\end{array}$ & $\begin{array}{c}\Delta(0) \\
\left(10^{-3} \mathrm{eV}\right)\end{array}$ & $\xi_{0}(\mu \mathrm{m})$ \\
\hline 52.72 & 53.67 & 6.21 & 7.12 & 1.07 & 1.42 & 1.51 & 1.08 & 0.28 \\
\hline
\end{tabular}

condition of "dirty alloys," $l \ll \xi_{0}$, which is characteristic of type II SCs. The coherence length, $\xi$, can be expressed as [3]:

$$
\xi=\left(\xi_{0} \ell\right)^{\frac{1}{2}} .
$$

The calculated $\xi$ of C5 is 206.1 Å, which is much larger than the value of YBCO (10 ̊̊) [23]. From Equation (12), the $\lambda$ of C5 is $2924 \AA \hat{A}$, so $\kappa(=\lambda / \xi) \sim 14.19$. Accordingly, the HEAs in this study are type II SCs.

\section{CONCLUSION}

Superconductivity was observed in NbTaTiZr-based HEAs with added Fe, Ge, Hf, Si, and/or V. The SC behavior of HEAs in this study depends on both the composition distribution of $\mathrm{Nb}+\mathrm{Ta}$ and the degree of lattice distortion that is caused by the change in the number of contained elements, $n$. As $n$ increases from four to seven, the $\mathrm{Nb}+\mathrm{Ta}$ content in the $\mathrm{SC}$ white phases falls, reducing $T_{c}$ In multiphase HEAs, the e/a ratio alone cannot be used to evaluate accurately $T_{\mathcal{o}}$ as in the manner of the Matthias rule. This study uses analysis of $R(T)$, and SQUID and Hall measurements to demonstrate that the HEAs in this study are type II SCs.

\section{ACKNOWLEDGEMENTS}

We acknowledge the financial support of the National Science Council, Taiwan, under No. 100-2221-E-007-110-MY3. 


\section{REFERENCES}

1. Onnes, H.K. (1913) Supplements to the Communications of the Physical Laboratory of Leiden University. 34b, 55.

2. Meissner, W. and Ochsenfeld, R. (1933) Ein neuer Effekt bei Eintritt der Supraleitfähigkeit. Naturwissenschaften, 21, 787-788. https://doi.org/10.1007/BF01504252

3. Dew-Hughes, D. (2001) The Critical Current of Superconductors: An Historical Review. Low Temperature Physics, 27, 713-722. https://doi.org/10.1063/1.1401180

4. Narasimhan, S.L., Taggart, R. and Polonis, D.H. (1972) The Superconducting Transition Behavior of ZrNb Binary Alloys. Journal of Nuclear Materials, 43, 258-268. https://doi.org/10.1016/0022-3115(72)90057-8

5. Cotton, W.L., Taggart, R. and Polonis, D.H. (1974) The Superconducting Transition Behavior of Ternary Alloys of Titanium. Scripta Metallurgica, 8, 329-336. https://doi.org/10.1016/0036-9748(74)90133-1

6. Matthias, B.T. (1957) Chap. 5 Superconductivity in the Periodic System. Progress in Low Temperature Physics, 2, 138-150. https://doi.org/10.1016/S0079-6417(08)60104-3

7. Yeh, J.W., Chen, S.K., Lin, S.J., Gan, J.Y., Tsau, T.S. and Chang, S.Y. (2004) Nanostructured High-Entropy Alloys with Multiple Principal Elements: Novel Alloy Design Concepts and Outcomes. Advanced Engineering. Materials, 6, 299-303. https://doi.org/10.1002/adem.200300567

8. Chen, S.K. (2012) Chap. 6 Electrochemical Passive Properties of AlxCoCrFeNi ( $\mathrm{x}=0,0.25,0.50,1.00)$ High-Entropy Alloys in Sulfuric Acids. In: Shih, H., Ed., Corrosion Resistance, InTech, Croatia, 133-156. https://doi.org/10.5772/33892

9. Tong, C.J., Chen, Y.L., Yeh, J.W., Lin, S.J., Chen, S.K., Shun, T.T., Tsau, C.H. and Chang, S.Y. (2005) Microstructure Characterization of $\mathrm{Al}_{\mathrm{x}} \mathrm{CoCrCuFeNi}$ High-Entropy Alloy System with Multiprincipal Elements. Metallurgical and Materials Transactions A, 36, 881-893. https://doi.org/10.1007/s11661-005-0283-0

10. Chen, S.K. and Kao, Y.F. (2012) Near-Constant Resistivity in $4.2-360 \mathrm{~K}$ in a $\mathrm{B} 2 \mathrm{Al}_{2.08} \mathrm{CoCrFeNi}$. AIP Advances, 2, 012111-012115. https://doi.org/10.1063/1.3679072

11. Kao, Y.F., Chen, S.K., Sheu, J.H., Lin, J.T., Lin, W.E., Yeh, J.W., Lin, S.J., Liou, T.H. and Wang, C.W. (2010) Hydrogen Storage Properties of Multi-Principal-Component CoFeMnTi $\mathrm{V}_{\mathrm{y}} \mathrm{Zr}_{\mathrm{z}}$ Alloys. International Journal of Hydrogen Energy, 35, 9046-9059.

12. Johnson, W.L. (1981) Superconducting Materials. In: Cahn, R.W. and Haasen, P., Eds., Physical Metallurgy, 2nd Edition, Elsevier, Amsterdam, Chap. 27, 1735-1778.

13. Bardeen, J., Cooper, L. and Schrieffer, J.R. (1957) Theory of Superconductivity. Physical Review Letters, 108, 1175.

14. McMillan, W.L. (1968) Transition Temperature of Strong-Coupled Superconductors. Physical Review B, 167, 331.

15. Das Gupta, A., Mordike, B.L. and Schultz, L. (1975) Superconductivity in a Dilute Copper-Niobium Alloy. Materials Science and Engineering, 18, 137-142.

16. Kittel, C. (2005) Introduction to Solid State Physics. 8th Edition, Wiley, Hoboken.

17. Khan, H.R. (2003) Superconductivity. In: Meyers, R.A., Ed., Encyclopedia of Physical Science and Technology, Elsevier, Amsterdam, 235-250.

18. Tsao, T.K. (2012) Nb-Containing Superconducting High-Entropy Alloys. M.S. Thesis, National Tsing Hua University, Hsinchu. (In Chinese)

19. Tiwari, G.P. and Ramanujan, R.V. (2001) Review the Relation between the Electron to Atom Ratio and Some Properties of Metallic Systems. Journal of Materials Science, 36, 271-283. 
20. Mota, A.C. and Hoyt, R.F. (1976) Dependence of the Superconducting Transition Temperature on Electron per Atom Ratio and Lattice Deformation in Noble Metal Alloys. Solid State Communications, 20, 1025-1028.

21. Ramakrishnan, S. and Chandra, G. (1985) Study of Superconductivity in Ternary $\mathrm{Nb}_{50} \mathrm{Ti}_{30} \mathrm{M}_{20}(\mathrm{M}=\mathrm{Mo}, \mathrm{Pt}, \mathrm{Pd}$, $\mathrm{V}$ and $\mathrm{Ru}$ ) Solid Solutions. Solid State Communications, 56, 965-966.

22. Bennett, L.H., Watson, R.E. and Carter, G.C. (1970) Relevance of Knight Shift Measurements Electronic Density of States. Journal of Research of the National Bureau of Standards A: Physics and Chemistry, 74, 569-610.

23. Cyrot, M. and Pavuna, D. (1992) Introduction to Superconductivity and High-T $\mathrm{T}_{\mathrm{c}}$ Materials. World Scientific, London. 